\title{
Faithful conversion of propagating quantum information to mechanical motion
}

\author{
A. P. Reed ${ }^{1,2 \star}$, K. H. Mayer ${ }^{2,3}$, J. D. Teufel ${ }^{3}$, L. D. Burkhart ${ }^{4}$, W. Pfaff ${ }^{4}$, M. Reagor ${ }^{4,5}$, L. Sletten ${ }^{1,2}$, \\ X. Ma ${ }^{1,2}$, R. J. Schoelkopf ${ }^{4}$, E. Knill ${ }^{3,6}$ and K. W. Lehnert ${ }^{1,2,3}$
}

The motion of micrometre-sized mechanical resonators can now be controlled and measured at the fundamental limits imposed by quantum mechanics. These resonators have been prepared in their motional ground state $^{1-3}$ or in squeezed states ${ }^{4-6}$, measured with quantum-limited precision ${ }^{7}$, and even entangled with microwave fields ${ }^{8}$. Such advances make it possible to process quantum information using the motion of a macroscopic object. In particular, recent experiments have combined mechanical resonators with superconducting quantum circuits to frequency-convert, store and amplify propagating microwave fields ${ }^{9-12}$. But these systems have not been used to manipulate states that encode quantum bits (qubits), which are required for quantum communication and modular quantum computation ${ }^{13,14}$. Here we demonstrate the conversion of propagating qubits encoded as superpositions of zero and one photons to the motion of a micromechanical resonator with a fidelity in excess of the classical bound. This ability is necessary for mechanical resonators to convert quantum information between the microwave and optical domains ${ }^{15-17}$ or to act as storage elements in a modular quantum information processor $12,13,18$. Additionally, these results are an important step towards testing speculative notions that quantum theory may not be valid for sufficiently massive systems ${ }^{19}$.

Quantum communication networks that use superconducting qubits and modular quantum computing architectures require the ability to store, amplify or frequency-shift propagating microwave fields. A single electromechanical device provides all of these functions by rapidly varying the parametric coupling between mechanical motion and microwave fields. For example, the ability to suddenly turn off the interaction between a microwave field and mechanical motion allows the state of a field propagating through a transmission line to be converted to, and trapped in, the motional state of the resonator ${ }^{12}$. To use this capture process in a general quantum information processor, one must work with states that have non-Gaussian statistics, such as qubits encoded as superpositions of zero and one photons. In contrast, any process using only Gaussian states can be simulated efficiently on a classical computer $^{20}$. But in the regime that most of these devices operate, the equations that describe the coupling are linear, ensuring that a Gaussian state of the microwave field or mechanical resonator will never evolve into a non-Gaussian state.

For electromechanical devices that manipulate propagating microwave fields, accessing non-Gaussian mechanical states requires either a source of non-Gaussian microwave fields or a nonlinear detector such as a single-photon counter. Forgoing the control of propagating fields, non-Gaussian mechanical states have been accessed by resonantly coupling a mechanical system to a qubit $^{1}$. More recent work has shown that parametrically coupling a mechanical resonator to a qubit via an intermediate cavity bus may enable access to non-Gaussian states ${ }^{21}$. But in this integrated device, the predicted transfer fidelity is low primarily because of the intrinsic loss in the cavity. So far, the demands of fabricating such hybrid devices have reduced the coherence of the mechanical resonator, qubit or cavity far below the state of the art.

In this work, we convert non-Gaussian states from propagating microwave fields to the motion of a micrometre-sized mechanical resonator. We use an electromechanical device to capture, store and amplify single photons generated by a superconducting qubit and then determine the density matrix of the mechanical resonator using quantum state tomography. We find that the quantum state

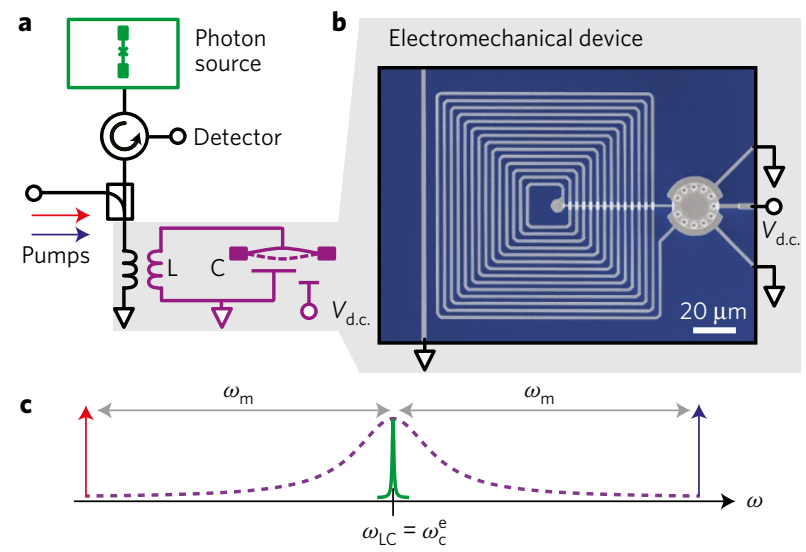

Figure 1 | Diagram of the experiment. a, A simplified schematic shows the electromechanical device connected to a photon source consisting of a transmon qubit in a microwave cavity. Pumps (arrows) used to create the capture (red) and amplification (blue) interactions are injected into the transmission line. b, False-colour micrograph of the electromechanical device where aluminium films (grey) are deposited on a sapphire substrate (blue). c, Pumps are detuned below and above the LC circuit's resonant frequency $\omega_{\mathrm{LC}}$ by the mechanical resonant frequency $\omega_{\mathrm{m}}$. Using a voltage bias $V_{\text {d.c., }}$ the LC circuit's resonant response (dashed magenta) is tuned to match the much narrower resonance of the microwave cavity (green solid) at the frequency $\omega_{\mathrm{c}}^{\mathrm{e}}$.

${ }^{1}$ JILA, Boulder, Colorado 80309-0440, USA. ${ }^{2}$ Department of Physics, University of Colorado, Boulder, Colorado 80309-0390, USA. ${ }^{3}$ National Institute of Standards and Technology (NIST), Boulder, Colorado 80305, USA. ${ }^{4}$ Departments of Applied Physics and Physics, Yale University, New Haven, Connecticut 06520, USA. ${ }^{5}$ Rigetti Computing, 775 Heinz Avenue, Berkeley, California 94710, USA. ${ }^{6}$ Center for Theory of Quantum Matter, University of Colorado, Boulder, Colorado 80309, USA. *e-mail: adam.reed@jila.colorado.edu 
can be stored on a timescale exceeding $100 \mu \mathrm{s}$, an improvement of over four orders of magnitude compared with previous work that demonstrated the storage of a non-Gaussian state in an electromechanical device ${ }^{1}$. To characterize how the capture process affects arbitrary propagating qubit states, we capture superpositions of zero and one photons. The degree to which this process preserves quantum information is quantified by the average fidelity ${ }^{22}$, which we find to be $F_{\text {avg }}=0.83_{-0.06}^{+0.03}$ where the limits are the $90 \%$ confidence interval. This level of performance exceeds the fidelity achievable using only classical resources, indicating that our electromechanical device is suitable for the transduction of quantum information.

The electromechanical device consists of an inductor-capacitor (LC) circuit that is tunable and coupled to a mechanical resonator (Fig. 1a). The tunability and coupling arise from the upper plate of the capacitor, which is a 100-nm-thick suspended and tensioned aluminium membrane that is free to vibrate. The fundamental drumhead-like vibrational mode of this membrane forms the mechanical resonance at $\omega_{\mathrm{m}} / 2 \pi \approx 9.3 \mathrm{MHz}$. If displaced by the resonator's zero-point motion of $6.4 \mathrm{fm}$, the circuit's resonant frequency shifts by $g_{0} / 2 \pi \approx 280 \mathrm{~Hz}$. The circuit also couples inductively to propagating microwave fields in a nearby transmission line at a rate of $\kappa_{\mathrm{LC}} / 2 \pi \approx 3 \mathrm{MHz}$. We tune the LC circuit into precise resonance with a narrow-band and fixedfrequency photon source by using a third electrode, biased at $V_{\text {d.c. }}$ relative to the membrane, to control the static separation between the membrane and the microwave electrode ${ }^{9}$.

We connect the electromechanical device to an on-demand source of single photons using the network depicted in Fig. 1a. To efficiently generate single photons compatible with the narrow bandwidth requirements ${ }^{12}$ of the electromechanical device, we use a circuit quantum electrodynamics system ${ }^{23}$. It consists of a transmon qubit with a transition frequency $\omega_{\mathrm{q}} / 2 \pi=$ $5.652 \mathrm{GHz}$ in a microwave cavity, whose resonance frequency is $\omega_{c}^{\mathrm{g}} / 2 \pi=7.290 \mathrm{GHz}$ when the qubit is in the ground state, $|g\rangle$, and $\omega_{c}^{\mathrm{e}} / 2 \pi=\left(\omega_{c}^{\mathrm{g}}-2 \chi\right) / 2 \pi=7.283 \mathrm{GHz}$ when it is in the excited state, $|e\rangle$, where $\chi$ is the dispersive shift. We use a control pulse ${ }^{24}$ to drive the transition $|g\rangle|0\rangle \rightarrow|e\rangle|1\rangle$, where $|0\rangle$ and $|1\rangle$ correspond to zero and one cavity photons, respectively. The cavity state then evolves into a field propagating through the transmission line with the centre frequency $\omega_{c}^{\mathrm{e}}$ and narrow bandwidth $\kappa_{\mathrm{c}} / 2 \pi=60 \mathrm{kHz}$.

The propagating microwave field is parametrically coupled to the membrane's motion by applying pumps to the LC circuit. To capture the state of the propagating field ${ }^{12}$, we use a pump that is detuned below (red-detuned) the LC resonance with detuning $\Delta_{\mathrm{r}}=-\omega_{\mathrm{m}}$. This pump creates an interaction that can exchange the states of the input microwave field and the mechanical resonator. For a given temporal envelope of the input field, the coupling, $\Gamma_{\mathrm{r}}(t)=4 g_{0}^{2} n_{\mathrm{r}}(t) / \kappa_{\mathrm{LC}}$, must be modulated for optimal capture efficiency ${ }^{9}$, where $n_{\mathrm{r}}(t)$ is the number of photons induced in the LC circuit by the pump. If instead we apply a bluedetuned pump at $\Delta_{\mathrm{b}}=+\omega_{\mathrm{m}}$, a two-mode squeezer interaction is created that amplifies both the motion of the resonator and the incident microwave field ${ }^{8}$. During amplification, the LC circuit emits a propagating field with a temporal envelope that rises exponentially at a rate of $\Gamma_{\mathrm{b}} / 2$, where $\Gamma_{\mathrm{b}}(t)=4 g_{0}^{2} n_{\mathrm{b}}(t) / \kappa_{\mathrm{LC}}$ is set by the average number of photons, $n_{\mathrm{b}}(t)$, induced in the circuit by the blue-detuned pump. Crucially, the state of the emitted field depends on both the states of the resonator and the input field before amplification ${ }^{25}$.

We exploit the parametric interactions in two protocols that are used to characterize the capture process. Because the process maps states at the input of the electromechanical device to the resonator, we must determine the input state and compare it with the captured state. To this end, we have developed 'calibration' and 'capture' protocols that enable us to determine the input and captured states, respectively (Fig. 2a). We initially test the two protocols
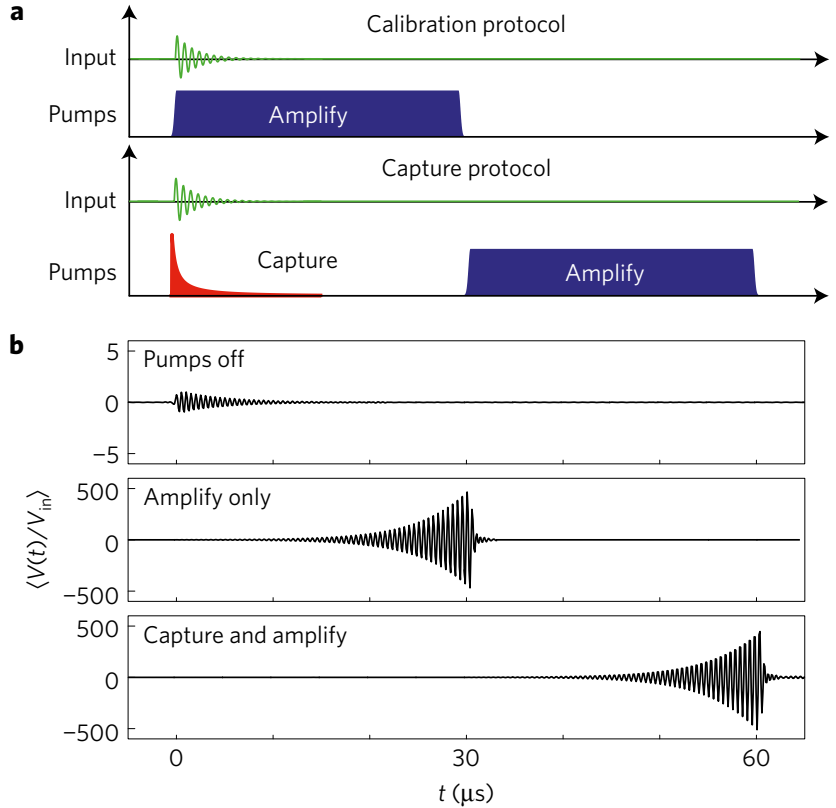

Figure 2 | Calibration and capture protocols. a, Timing diagrams depicting the input coherent signal (green) of amplitude $V_{\text {in }}$ and the pulse shapes of the pumps, $\Gamma_{\mathrm{r}}(t)$ and $\Gamma_{\mathrm{b}}(t)$, used to create the capture or amplification interaction. For the calibration protocol (top), the amplification pump is coincident with the signal pulse. The capture protocol (bottom) has a timing diagram similar to the calibration protocol, but the input signal is coincident with a capture pulse that is temporally shaped for optimal capture of the signal. At $t=30 \mu \mathrm{s}$, the mechanical state is amplified and converted back into a microwave field. $\mathbf{b}$, The plots show the voltage signals, $V(t)$, measured at the detector and averaged over 500 repetitions of each protocol when the pumps were either off or on. During amplification, $\Gamma_{\mathrm{b}} / 2 \pi=60 \mathrm{kHz}$, which results in a gain of $53 \mathrm{~dB}$.

with coherent signals whose frequency and bandwidth are chosen to match those created by the circuit quantum electrodynamics system (Fig. 2b).

In the calibration protocol, the input field is amplified directly and then measured. We implement this protocol by applying the blue-detuned pump coincident with the input field. In this case, the electromechanical device functions as a linear phase-preserving amplifier whose input and output are the incident and reflected microwave fields, respectively. These pulsed fields have different envelopes; nevertheless, with an appropriate filter (Supplementary Information) they are related by an energy gain of $\cosh ^{2}(r / 2)$, where $r=\Gamma_{\mathrm{b}} \tau_{\mathrm{b}}$ and $\tau_{\mathrm{b}}$ is the pump's duration. If we regard the input of the amplifier as the incident microwave field, the fluctuations of the resonator's motion are the source of the amplifier's added noise, reaching the quantum $\operatorname{limit}^{25}$ if the resonator is in its ground state ${ }^{2}$.

After obtaining the input state, we use the capture protocol to determine the resonator state. We first apply the red-detuned pump coincident with the input field. Once it is captured, we then apply the blue-detuned pump to amplify the resonator's state. In contrast to the calibration protocol, we now regard the amplifier's input to be the state of the resonator. The output is still the reflected field, but the added noise is due to the vacuum fluctuations of the incident field. When interpreted this way, we realize a linear phase-conjugating amplifier with an energy gain of $\sinh ^{2}(r / 2)$.

Operating the electromechanical device as a low-noise amplifier enables us to perform state tomography on both the input microwave field and on the motion of the resonator. For each repetition of the two protocols depicted in Fig. 3, we record a voltage signal, $V(t)$, at the detector during amplification. For each 
a

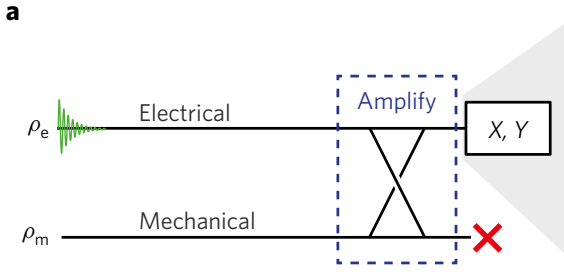

d

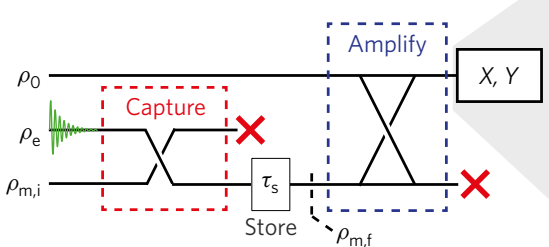

b

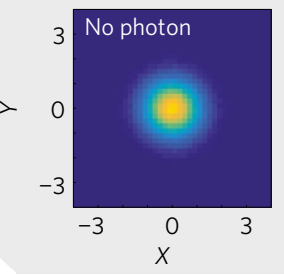

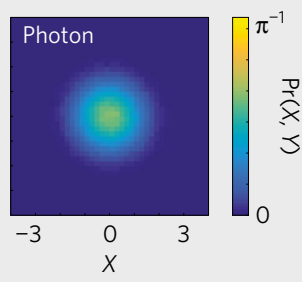

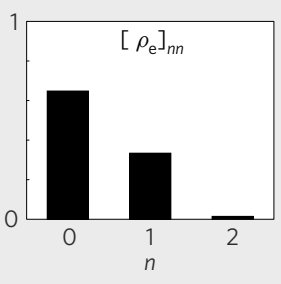

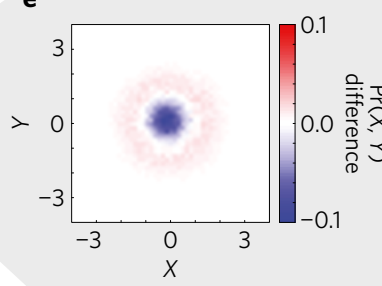

f

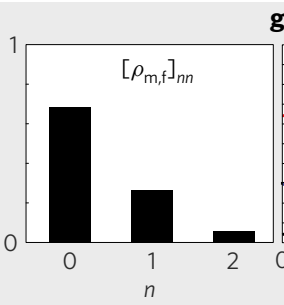

g

Figure 3 | Capture, storage and amplification of single propagating photons. a, The diagram shows the calibration protocol where the green decaying sinusoid represents an input microwave field in the state $\rho_{\mathrm{e}}$. Prior to injecting an input state, the mechanical mode described by $\rho_{\mathrm{m}}$ is cooled close to its quantum ground state. A red cross indicates a mode is not measured. In modelling the density matrix of the measured mode, we trace over these unmeasured modes. b. Histograms of 512,000 measured quadrature amplitudes $X$ and $Y$ for the input state are plotted such that the histograms form a discretized and normalized joint probability distribution $\operatorname{Pr}(X, Y)$. The labels 'no photon' and 'photon' indicate whether single photons were generated or not. c, The diagonal elements of $\rho_{\mathrm{e}}$ are obtained using a method of maximum likelihood state tomography (Supplementary Information). d, The diagram shows the capture protocol where $\rho_{\mathrm{m}, \mathrm{i}}$ is the initial mechanical state and $\tau_{\mathrm{s}}$ is an adjustable storage time. After storage, the mechanical mode is in the final state $\rho_{\mathrm{m}, \mathrm{f}}$. During amplification, the electrical mode is in a vacuum state $\rho_{0}$. e, The figure shows the difference of the 'photon' and 'no photon' histograms acquired using the capture protocol, highlighting the phase-symmetric character of a single-phonon state. f, Diagonal elements of $\rho_{\mathrm{m}}$ obtained at $\tau_{\mathrm{s}}<3 \mu \mathrm{s}$. $\mathbf{g}$, The diagonal elements of $\rho_{\mathrm{m}}$ decay toward their thermal equilibrium values as a function of $\tau_{\mathrm{s}}$. A model (solid lines) of $\rho_{\mathrm{m}}$ yields a characteristic storage time of $\tau_{\mathrm{m}}=137 \pm 6 \mu \mathrm{s}$ (Supplementary Information).

a
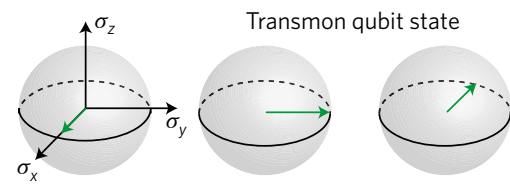

Mechanical quadrature amplitudes

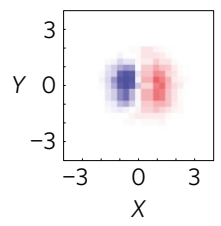

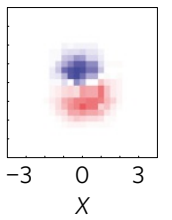

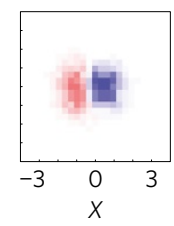

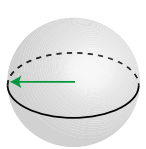

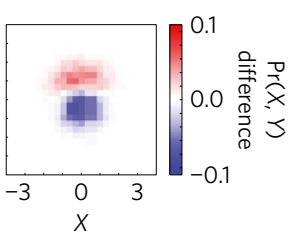

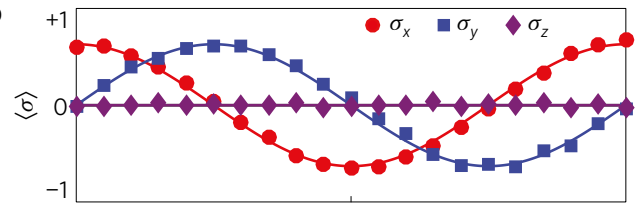

d

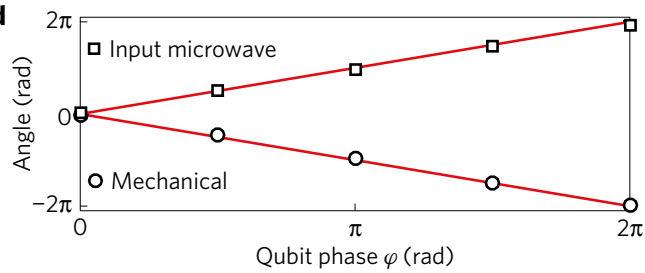

Figure 4 | Conversion of propagating qubits. a, The transmon qubit was prepared in the superposition state $(1 / \sqrt{2})\left(|g\rangle+e^{i \varphi}|e\rangle\right)$ with a phase $\varphi$ chosen from the set $\{0, \pi / 2, \pi, 3 \pi / 2\}$, as denoted by the green arrow plotted on a Bloch sphere. $\mathbf{b}$, The plot shows measured Pauli component amplitudes, $\left\langle\sigma_{k}\right\rangle$, of the qubit state as a function of its phase where $k=\{x, y, z\}$. Readout of the qubit state was achieved by detecting the qubit-state-dependent shift of the cavity and using the electromechanical device as a microwave amplifier (Supplementary Information). The solid lines are fits with the readout contrast of $60 \%$ as the only free parameter. c, Subtracted histograms (similar to Fig. 3e) of 20,480 measurements show the mechanical quadrature amplitudes, $X$ and $Y$, as the transmon qubit's phase was varied. $\mathbf{d}$, The plot shows the argument of the off-diagonal density matrix element, $\rho_{01}$, for both the input microwave, $\rho_{\mathrm{e}}$, and captured mechanical, $\rho_{\mathrm{m}}$, states as a function of $\varphi$. The mechanical state changes linearly in $\varphi$, indicating that the conversion process is coherent. The apparent opposite dependence of $\varphi$ of the input and captured states is a result of the phase-conjugate amplification of the mechanical state compared with the direct amplification of the input microwave state (solid lines indicate the expected behaviour).

voltage record, we extract a pair of quadrature amplitudes, $X$ and $Y$, for the state of either the resonator or input field (Supplementary Information). By making repeated measurements of $V(t)$, we obtain a set of quadrature amplitudes and use this information to extract a density matrix $\rho$ via a method of maximum likelihood state tomography ${ }^{26}$ (Supplementary Information). We refer to the states of the input microwave field and of the mechanical resonator as $\rho_{\mathrm{e}}$ and $\rho_{\mathrm{m}}$, respectively.

To test the conversion of non-Gaussian states, we inject single photons into the electromechanical device. We also operate the calibration and capture protocols (Fig. 3a,d) without generating single photons. In this case, we inject a vacuum state to determine the gain of the detector which we use to scale $X$ and $Y$ in units of (quanta) ${ }^{1 / 2}$ (Supplementary Information). Prior to the execution of each protocol, we cool the resonator close to its quantum ground state $^{2}$ with an occupancy of approximately 0.1 quanta (Supplementary Information). For both protocols, the tomography yields density matrix estimates containing significant elements only on the diagonals (Fig. 3c,f). In particular, we find that the probability of detecting a single photon is $\left[\rho_{\mathrm{e}}\right]_{11}=0.33_{-0.01}^{+0.02}$ (Supplementary Information). After capture, the probability of a single phonon occupying the mechanical mode is $\left[\rho_{\mathrm{m}}\right]_{11}=0.26_{-0.02}^{+0.01}$. To distinguish 
the captured state from a thermal or coherent state, we calculate the degree of second-order coherence $g_{\mathrm{m}}^{(2)}=0.89_{-0.17}^{+0.05}$ (Supplementary Information). For comparison, a thermal or coherent state of motion yields $g_{\mathrm{m}}^{(2)} \geq 1$. After capturing single photons, we vary the storage time $\tau_{\mathrm{s}}$ and test the ability to mechanically store a non-Gaussian state (Fig. 3g). We use a master equation formalism to model the evolution of $\rho_{\mathrm{m}}$ with the characteristic storage time $\tau_{\mathrm{m}}$ as the only free parameter (Supplementary Information). We extract $\tau_{\mathrm{m}}=137 \pm 6 \mu \mathrm{s}$, which is about ten times longer than the time used to capture the input photon state.

Having demonstrated the ability to capture single photons, we then characterize how the capture process affects arbitrary qubit states encoded as superpositions of zero and one photons. This process is described by a map $\mathcal{E}$ between incident and captured states whose quality is characterized by the average fidelity ${ }^{22}$

$$
F_{\text {avg }}=\int \mathrm{d} \Psi\langle\Psi|\mathcal{E}(|\Psi\rangle\langle\Psi|)| \Psi\rangle
$$

which measures how indistinguishable the output of the process is from the input, averaged over all pure input states $|\Psi\rangle$. To determine $F_{\text {avg }}$, it is sufficient to capture a set of states that includes a singlephoton state and superpositions of zero and one photons. We can create superposition states by first preparing the transmon qubit in the superposition $(1 / \sqrt{2})\left(|g\rangle+\mathrm{e}^{i \varphi}|e\rangle\right)$, with varying phase $\varphi$, as shown in Fig. 4a,b. By driving the transition $|g\rangle|0\rangle \rightarrow|e\rangle|1\rangle$, we transfer the superposition state from the transmon to the cavity and then let the cavity state evolve into the propagating field. Operating the capture protocol on this set of states shows that the phase of the qubit state is converted to the motion of the mechanical resonator (Fig. 4c). More quantitatively, we follow the procedure illustrated in Fig. 3, determining both $\rho_{\mathrm{e}}$ and $\rho_{\mathrm{m}}$ for this set of states (Fig. 4d). From the input and output density matrices, we calculate for arbitrary qubit states $F_{\text {avg }}=0.83_{-0.06}^{+0.03}$, which is consistent with a cascaded beamsplitter model of the capture process (Supplementary Information). Crucially, the average fidelity exceeds $2 / 3$, the highest possible fidelity for transferring qubits using only classical resources (Supplementary Information).

Converting microwave qubit states to mechanical motion opens up new possibilities to process quantum information using micrometre-sized mechanical resonators. To communicate quantum information between remote modules in a network, such resonators may be the key element in the transduction of microwave quantum signals to telecommunications light ${ }^{15-17}$. For quantum computation protocols that require the feed-forward of information, such as teleportation ${ }^{27}$ and error correction schemes ${ }^{28}$, mechanical resonators can act as on-demand memories for quantum states. As microfabrication advances continue to reduce mechanical dissipation, it could become possible to store a quantum state in the motion of a macroscopic object for about one minute ${ }^{29,30}$.

Data availability. The data that support the plots within this paper and other findings of this study are available from the corresponding author upon reasonable request.

Received 31 January 2017; accepted 9 August 2017; published online 18 September 2017

\section{References}

1. O'Connell, A. D. et al. Quantum ground state and single-phonon control of a mechanical resonator. Nature 464, 697-703 (2010).

2. Teufel, J. D. et al. Sideband cooling of micromechanical motion to the quantum ground state. Nature 475, 359-363 (2011).

3. Chan, J. et al. Laser cooling of a nanomechanical oscillator into its quantum ground state. Nature 478, 89-92 (2011).

4. Wollman, E. E. et al. Quantum squeezing of motion in a mechanical resonator. Science 349, 952-955 (2015).
5. Lecocq, F., Clark, J. B., Simmonds, R. W., Aumentado, J. \& Teufel, J. D. Quantum nondemolition measurement of a nonclassical state of a massive object. Phys. Rev. X 5, 041037 (2015).

6. Pirkkalainen, J. M., Damskägg, E., Brandt, M., Massel, F. \& Sillanpää, M. A. Squeezing of quantum noise of motion in a micromechanical resonator. Phys. Rev. Lett. 115, 243601 (2015).

7. Teufel, J. D., Donner, T., Castellanos-Beltran, M. A., Harlow, J. W. \& Lehnert, K. W. Nanomechanical motion measured with an imprecision below that at the standard quantum limit. Nat. Nanotech. 4, 820-823 (2009).

8. Palomaki, T. A., Teufel, J. D., Simmonds, R. W. \& Lehnert, K. W. Entangling mechanical motion with microwave fields. Science 342, 710-713 (2013).

9. Andrews, R. W., Reed, A. P., Cicak, K., Teufel, J. D. \& Lehnert, K. W. Quantum-enabled temporal and spectral mode conversion of microwave signals. Nat. Commun. 6, 10021 (2015).

10. Lecocq, F., Clark, J. B., Simmonds, R. W., Aumentado, J. \& Teufel, J. D. Mechanically mediated microwave frequency conversion in the quantum regime. Phys. Rev. Lett. 116, 043601 (2016).

11. Ockeloen-Korppi, C. F. et al. Low-noise amplification and frequency conversion with a multiport microwave optomechanical device. Phys. Rev. X 6, 041024 (2016)

12. Palomaki, T. A., Harlow, J. W., Teufel, J. D., Simmonds, R. W. \& Lehnert, K. W. Coherent state transfer between itinerant microwave fields and a mechanical oscillator. Nature 495, 210-214 (2013).

13. Monroe, C. et al. Large-scale modular quantum-computer architecture with atomic memory and photonic interconnects. Phys. Rev. A 89, 022317 (2014).

14. Narla, A. et al. Robust concurrent remote entanglement between two superconducting qubits. Phys. Rev. X 6, 031036 (2016).

15. Bochmann, J., Vainsencher, A., Awschalom, D. D. \& Cleland, A. N. Nanomechanical coupling between microwave and optical photons. Nat. Phys. 9, 712-716 (2013).

16. Andrews, R. W. et al. Bidirectional and efficient conversion between microwave and optical light. Nat. Phys. 10, 321-326 (2014).

17. Fang, K., Matheny, M. H., Luan, X. \& Painter, O. Optical transduction and routing of microwave phonons in cavity-optomechanical circuits. Nat. Photon. 10, 489-496 (2016).

18. Pfaff, W. et al. Controlled release of multiphoton quantum states from a microwave cavity memory. Nat. Phys. http://dx.doi.org/10.1038/nphys4143 (2017).

19. Arndt, M. \& Hornberger, K. Testing the limits of quantum mechanical superpositions. Nat. Phys. 10, 271-277 (2014).

20. Bartlett, S. D., Sanders, B. C., Braunstein, S. L. \& Nemoto, K. Efficient classical simulation of continuous variable quantum information processes. Phys. Rev. Lett. 88, 097904 (2002).

21. Lecocq, F., Teufel, J. D., Aumentado, J. \& Simmonds, R. W. Resolving the vacuum fluctuations of an optomechanical system using an artificial atom. Nat. Phys. 11, 635-639 (2015).

22. Nielsen, M. A. A simple formula for the average gate fidelity of a quantum dynamical operation. Phys. Lett. A 303, 249-252 (2002)

23. Paik, H. et al. Observation of high coherence in Josephson junction qubits measured in a three-dimensional circuit QED architecture. Phys. Rev. Lett. 107, 240501 (2011).

24. Kindel, W. F., Schroer, M. D. \& Lehnert, K. W. Generation and efficient measurement of single photons from fixed-frequency superconducting qubits. Phys. Rev. A 93, 033817 (2016).

25. Caves, C. M., Combes, J., Jiang, Z. \& Pandey, S. Quantum limits on phase-preserving linear amplifiers. Phys. Rev. A 86, 063802 (2012).

26. Eichler, C. et al. Experimental state tomography of itinerant single microwave photons. Phys. Rev. Lett. 106, 220503 (2011).

27. Bennett, C. H. et al. Teleporting an unknown quantum state via dual classical and Einstein-Podolsky-Rosen channels. Phys. Rev. Lett. 70, 1895-1899 (1993)

28. Ofek, N. et al. Extending the lifetime of a quantum bit with error correction in superconducting circuits. Nature 536, 441-445 (2016).

29. Norte, R. A., Moura, J. P. \& Gröblacher, S. Mechanical resonators for quantum optomechanics experiments at room temperature. Phys. Rev. Lett. 116, 147202 (2016).

30. Reinhardt, C., Müller, T., Bourassa, A. \& Sankey, J. C. Ultralow-noise SiN trampoline resonators for sensing and optomechanics. Phys. Rev. X 6, 021001 (2016).

\section{Acknowledgements}

We acknowledge advice from C. Axline, M. Castellanos-Beltran, L. Frunzio, S. Glancy, W. F. Kindel and F. Lecocq as well as technical assistance from R. Delaney and H. Greene. We thank P. Blanchard for assistance in taking the micrograph shown in Fig. 1b. We acknowledge funding from the National Science Foundation (NSF) under grant number 1125844, AFOSR MURI under grant number FA9550-15-1-0015, and the Gordon and 
Betty Moore Foundation. A.P.R. acknowledges support from the NSF Graduate Research Fellowship under grant number DGE 1144083. L.D.B. acknowledges the support of the ARO QuaCGR Fellowship.

\section{Author contributions}

A.P.R. and K.W.L. designed the experiment. A.P.R. performed the measurements. K.H.M designed the tomographic analysis. A.P.R., K.H.M., J.D.T., E.K. and K.W.L. analysed the results. A.P.R., K.H.M. J.D.T and K.W.L. wrote the manuscript. L.D.B. and A.P.R fabricated the devices. A.P.R., L.D.B., W.P., M.R., L.S., X.M. and R.J.S. designed and constructed the photon source.

\section{Additional information}

Supplementary information is available in the online version of the paper. Reprints and permissions information is available online at www.nature.com/reprints. Publisher's note: Springer Nature remains neutral with regard to jurisdictional claims in published maps and institutional affiliations. Correspondence and requests for materials should be addressed to A.P.R.

\section{Competing financial interests}

The authors declare no competing financial interests. 